\title{
La privación desde un enfoque multidimensional: el caso brasileño
}

\author{
Ana Flavia Machado, Andre Braz Golgher \\ y Mariangela Furlan Antigo
}

RESUMEN

Sobre la base del enfoque de las capacidades, en este trabajo se analiza la multidimensionalidad de la privación en las zonas urbanas del Brasil entre 2003 y 2008. El uso de un índice de cuatro dimensiones (condiciones de vivienda, salud, niveles de educación y participación en el mercado de trabajo) basadas en 13 indicadores revela que la mayoría de las personas vivían en hogares sin privación o en los que esta se registraba respecto de un solo indicador. Al comparar los resultados con el índice de privación según los niveles de ingreso en los estados del Brasil se confirma la desigualdad regional en ambos tipos de medición. Por último, a partir de datos de cohortes sintéticas y modelos de mínimos cuadrados ordinarios (MCO), se verifica la relación entre los atributos personales y la propensión a caer en la privación y a mantenerse en ellas.

PALABRAS CLAVE

CLASIFICACIÓN JEL

AUTORES
Zonas urbanas, condiciones de vida, pobreza, indicadores económicos, análisis comparativo, desigualdades regionales, Brasil

I31, I32

Ana Flavia Machado es profesora titular del Centro de Desarrollo y Planificación Regional (cedeplar) de la Universidad Federal de Minas Gerais (UfmG). afmachad@cedeplar.ufmg.br

Andre Braz Golgher es profesor asociado del Centro de Desarrollo y Planificación Regional (CedePLAR) de la Universidad Federal de Minas Gerais (UFMG). agolgher@cedeplar.ufmg.br

Mariangela Furlan Antigo es profesora asociada del Centro de Desarrollo y Planificación Regional (cedeplar) de la Universidad Federal de Minas Gerais (UfMG). maantigo@cedeplar.ufmg.br 


\section{I}

\section{Introducción}

Con el enfoque de las capacidades se introdujeron nuevos conceptos en la agenda de la investigación relacionada con el bienestar (Sen, 1999). Los conceptos de ese enfoque, entre ellos los de funcionamientos, capacidades $\mathrm{y}$ condiciones del agente, ampliaron el campo de estudio de la privación y permitieron superar las limitaciones de los indicadores tradicionales, basados principalmente en las nociones de activos, consumo, ingresos y utilidad.

En algunos estudios recientes se analizan los datos brasileños desde una perspectiva multidimensional basada en el enfoque de las capacidades. Por ejemplo, D'Ambrosio y Rodrigues (2008) utilizaron una medida no monetaria con diferentes indicadores de bienestar individual, y Neri (2008) construyó un índice de desarrollo humano (IDH) con variables relacionadas con la salud, la alimentación, los ingresos, la situación económica y política actual y el mercado de trabajo, entre otras. Bagolin y Ávila (2006) y Lopes, Macedo y Machado (2003 y 2004) midieron la privación desde una perspectiva multidimensional basada en la teoría de los conjuntos difusos. Silva y Barros (2006) y Rocha, Moreira y Santos (2008) también crearon un índice de privación multidimensional con respecto a las familias, las regiones y a grupos específicos de la población. Golgher (2010a) analizó la privación percibida por las familias brasileñas y observó que los perfiles de privación difieren en gran medida según se trate de áreas urbanas o rurales. Este autor también mostró que existe una escasa correlación entre privación e ingresos en la mayoría de las dimensiones consideradas en el estudio (Golgher, 2010b).

Si bien el propósito de este trabajo también consiste en examinar la multidimensionalidad de la privación en el Brasil sobre la base del enfoque de las capacidades, los objetivos establecidos y la metodología aplicada son diferentes. En este sentido, se construyó un índice de privación multidimensional (IPM) inspirado en la metodología propuesta por Alkire y Foster (2007) y aplicada en 104 países en desarrollo, incluido el Brasil, por Alkire y Santos (2010). El IPM está formado por cuatro dimensiones (condiciones de vivienda, salud, educación y mercado de trabajo), basadas en 13 indicadores no monetarios (véase el cuadro 2). Se observa que la mayoría de las personas viven en hogares sin privación o en los que esta se registra en relación con un solo indicador. Los hogares con carencias en muchos indicadores representan una pequeña proporción del total.
A continuación, se compara la privación medida por niveles de ingreso con el IPM en 2003 y 2008. El análisis regional de los datos mediante mapas revela una considerable heterogeneidad espacial entre los estados brasileños. La verificación de perfiles espaciales muy diferentes muestra que, como señalan Baulch y Masset (2003), los indicadores de privación monetarios y no monetarios no están correlacionados en los países en desarrollo. Estos resultados iniciales permiten una visión más general de la privación en el Brasil, tanto desde una perspectiva monetaria como multidimensional.

En el análisis se tuvieron en cuenta las regularidades y relaciones entre los 13 indicadores del IPM. El indicador relativo al servicio sanitario o sistema de disposición de las aguas residuales (alcantarillado) fue el más significativo en el Brasil, tanto desde el punto de vista unidimensional como multidimensional. Algunos aspectos de la privación pueden manifestarse en grupos de dos o más indicadores, como por ejemplo, agua y alcantarillado, trabajo infantil e inasistencia escolar y presencia de activos, adultos sin trabajo y analfabetos funcionales en el hogar. Estos resultados se confirmaron mediante un análisis factorial.

El presente artículo se divide en siete secciones, incluida esta Introducción en la que se contextualiza el tema del trabajo. En la segunda sección se describen la metodología aplicada para la construcción del índice de privación multidimensional (IPM), la base de datos utilizada y las dimensiones de la privación. En las cuatro secciones siguientes se presentan los resultados obtenidos a partir del IPM. Cada una de ellas tiene un objetivo específico: mientras que en la tercera se entrega una visión general del índice y su comparación con la privación monetaria, en las demás se muestran diferentes perspectivas del IPM. Así, en la cuarta sección se incluyen los resultados relativos a los estados brasileños, con el objetivo de evidenciar que los indicadores presentan diferencias regionales y escasa correlación. En la quinta sección se analizan algunas especificidades de cada dimensión de la privación, sus regularidades y relaciones, y en la sexta se aborda la dinámica de la privación mediante el uso de cohortes sintéticas y la aplicación del modelo de mínimos cuadrados ordinarios (MCO). Con ello se pretende dar una visión integral de la privación en el Brasil urbano, desde una perspectiva dinámica multidimensional. En la séptima y última sección se plantean algunas consideraciones y conclusiones. 


\section{II \\ Índice de privación multidimensional (IPM), datos e indicadores}

De acuerdo con el enfoque de las capacidades, las diferencias entre las personas determinan el grado de conversión de recursos en capacidades y estas no pueden medirse por los recursos disponibles, sino por lo que las personas pueden ser y hacer con ellos. Como propuso Sen (2001), se asume la naturaleza incompleta del enfoque de las capacidades para mantener su objetivo básico: la libertad de los investigadores para elegir y señalar los funcionamientos y las capacidades que han de tenerse en cuenta.

A partir de esas premisas, se utilizaron los datos de la Encuesta Nacional de Hogares (PNAD por sus siglas en portugués) de 2003 y 2008 y sus suplementos especiales. Si bien se trata de datos muy completos, no fueron creados con el objetivo de describir las percepciones de las personas sobre la privación. Por ese motivo, se optó por aplicar una metodología similar a la propuesta por Alkire y Foster (2007). A continuación se presentan brevemente la metodología y la construcción del índice de privación utilizado en este trabajo, así como la base de datos, las dimensiones y los indicadores empleados en la construcción del índice.

\section{Revisión del índice de privación multidimensional (IPM)}

La construcción del índice de privación relativo al Brasil se basó en la metodología propuesta por Alkire y Foster (2007), que se aplicó a diversos países en desarrollo en Alkire y Santos (2010). En primer lugar, se definen las principales dimensiones relativas a los funcionamientos y capacidades, a saber: condiciones de vivienda, salud, educación y participación en el mercado de trabajo ${ }^{1}$. Estas dimensiones se dividieron en 13 indicadores, que se detallan en el cuadro 1.

Las condiciones de vivienda se relacionan con el acceso al servicio sanitario y al agua por cañería, el destino de los residuos domiciliarios, el tipo de combustible utilizado para cocinar, la presencia de bienes en el hogar (activos), el material de las paredes de la vivienda y un

\footnotetext{
${ }^{1}$ Alkire y Santos (2010) definen las dimensiones sobre la base de los Objetivos de Desarrollo del Milenio. Estos autores utilizan una base de datos secundaria, como la que se empleó en este trabajo.
}

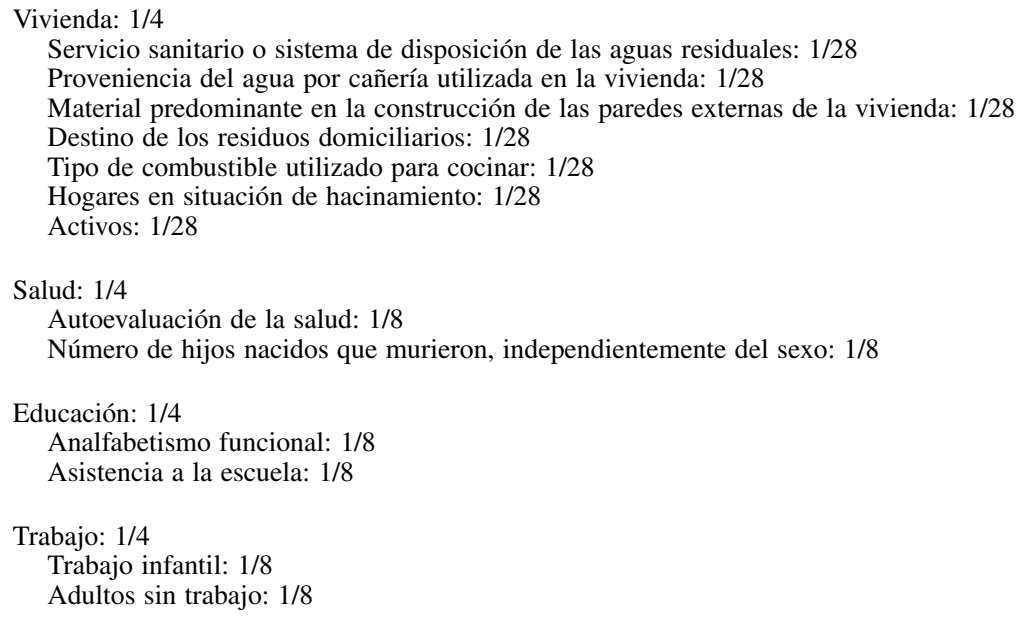

Fuente: elaboración propia.

IPM: índice de privación multidimensional. 
indicador para hogares en situación de hacinamiento. Los indicadores de salud son la autoevaluación de la salud y la muerte de hijos nacidos vivos en el hogar. El analfabetismo funcional y la asistencia escolar constituyen los indicadores de educación. La dimensión que se refiere al mercado de trabajo está compuesta por dos indicadores: incidencia del trabajo infantil y adultos sin trabajo en el hogar. Estos indicadores se describen con más detalle en la próxima sección.

Una vez definidas las dimensiones y los indicadores que componen el IPM, es necesario establecer la importancia de cada uno de ellos. Decancq y Lugo (2010) comparan ocho enfoques para la definición de grados de importancia en los índices multidimensionales. La categorización puede orientarse por medio de la distribución de los datos en la base de datos, pudiendo ser normativa o una combinación de ambos enfoques. Siguiendo esa dirección, Alkire y Foster (2007) argumentan que la importancia de cada dimensión debe definirse de acuerdo con criterios normativos (derechos humanos, convenciones internacionales, legislación nacional, consenso político, entre otros) y, dentro de cada dimensión, la orientación puede ser normativa mediante la distribución de los datos. Entre los criterios normativos, se puede optar por atribuir grados de importancia iguales o valores arbitrarios (Decancq y Lugo, 2010).

Alkire y Santos (2010) adoptaron un criterio de uniformidad entre las dimensiones y dentro de ellas a partir de una base de datos organizada con fines distintos a la captación del concepto de capacidades en sentido estricto. Siguiendo la propuesta de estos autores, se optó por atribuir la misma importancia a cada dimensión (1/4) y, dentro de cada dimensión, grados de importancia igualmente distribuidos entre los indicadores (véase el cuadro 1).

Se realizaron estimaciones de cada indicador con respecto a los hogares. Cuando el valor del indicador está por debajo de un umbral específico, las personas que viven en esos hogares se clasifican como pobres y, en caso contrario, como no pobres. De esa forma, el hogar puede clasificarse como no pobre o como sujeto a privación en por lo menos uno de los 13 indicadores considerados.

Alkire y Foster (2007) proponen ajustar la proporción de personas pobres clasificadas en la privación en cada indicador $(\mathrm{H})$ con una medida de intensidad de privación. Los autores definen la distancia normalizada (A) como la distancia entre el valor observado y un valor límite arbitrario definido para cada indicador. $\mathrm{Si}$ el valor observado es igual o superior al umbral de privación, la brecha normalizada asume el valor cero
(0). La normalización se realiza para poder ordenar los indicadores y compararlos.

De esta forma, IPM definido por Alkire y Santos (2010) está dado por:

$$
\mathrm{IPM}=\mathrm{H} * \mathrm{~A}
$$

\section{Fuentes de datos e indicadores}

Las fuentes de datos utilizadas fueron la Encuesta Nacional de Hogares de 2003 y de 2008 y sus suplementos especiales, que incluyen información sobre las condiciones de vida y de salud. Se utilizaron datos individuales y de los hogares y los indicadores se estimaron para esta última unidad.

Los datos se refieren solo al área urbana, pues los indicadores de algunas dimensiones difieren en gran medida según se trate de áreas urbanas o rurales y eso dificulta la definición de una única línea de privación para ambas. Por ejemplo, mientras que una vivienda situada en el área rural no debe, en principio, considerarse pobre si no tiene acceso a la red general de abastecimiento de agua, no puede decirse lo mismo con respecto al área urbana. Al tratar la privación a nivel de los hogares, se excluyó a las personas que indicaron ser pensionistas, empleados domésticos o familiares de un empleado doméstico. La muestra, sin expansión, es de 289.766 personas en 2003 y de 312.872 personas en 2008.

Como se aprecia en el cuadro 1, el IPM tiene cuatro dimensiones: condiciones de vivienda, salud, educación y trabajo. Las tres primeras guardan relación directa con el índice propuesto por Alkire y Santos (2010), adaptado a los datos utilizados en este trabajo, mientras que la última se incluyó debido a las especificidades del Brasil.

Como se detalla en el cuadro 2, la primera dimensión, relativa a las condiciones de la vivienda, abarca siete indicadores. El valor $(0)$ se atribuye a las personas que residen en hogares sin privación en la medida del indicador y el valor (1) se refiere a aquellas que sufren privación. El acceso a servicios públicos de disposición de las aguas residuales, agua por cañería y recolección de los residuos domiciliarios contribuye a valorizar la vivienda, pero sobre todo a reducir la morbilidad y mortalidad de los residentes. Entre las condiciones de la vivienda también se tienen en cuenta la presencia de cocina a gas o eléctrica, el acceso a bienes privados (teléfono móvil celular, radio, televisión a color, refrigerador de una o dos puertas) y el material de cobertura de las paredes. Con estas variables se procura evidenciar la capacidad de los residentes para satisfacer las necesidades básicas de la vida, incluida la de mantenerse informados, y el 


\section{Descripción de los indicadores del IPM por dimensiones}

A. Condiciones de la vivienda

A.1 Servicio sanitario o sistema de disposición de las aguas residuales

(1) Fosa séptica no conectada a la red de alcantarillado o pluvial, fosa rudimentaria, zanja, directo al río, lago o mar u otras

(0) Red de alcantarillado o pluvial y fosa séptica conectada a la red de alcantarillado o pluvial

A.2 Proveniencia del agua por cañería utilizada en la vivienda

(1) Pozo o manantial u otras

(0) Red general de distribución

A.3 Destino de los residuos domiciliarios

(1) Recolectados indirectamente, quemados o enterrados en la propiedad, arrojados en terreno baldío o espacio público, arrojados al río, lago o mar u otro destino

(0) Recolectados directamente

A.4 Tipo de combustible utilizado para cocinar

(1) Leña, carbón, energía eléctrica u otro combustible

(0) Gas envasado o por cañería

A.5 Activos

(1) Ninguno o solo uno de los siguientes activos en el hogar: teléfono móvil celular, radio, televisión a color, refrigerador de una o dos puertas

(0) Por lo menos dos activos

A.6 Material predominante en la construcción de las paredes externas de la vivienda

(1) Tapia no revestida, madera aprovechada, paja u otro material

(0) Albañilería o madera aserrada

A.7 Hogares en situación de hacinamiento

(1) Más de tres habitantes por dormitorio

(0) Hasta dos habitantes por dormitorio

B. Salud

B.1 Autoevaluación de la salud

(1) Si por lo menos un habitante declara que su salud es muy mala/mala

(0) Si todos los residentes consideran que su estado de salud es muy bueno, bueno o regular

B.2 Número de hijos nacidos que murieron, independientemente del sexo

(1) 1 o más

(0) Cero

\section{Educación}

C.1 Analfabetismo funcional

(1) 1 o más personas de 14 años o más que poseen 3 años o menos de estudio

(0) No hay personas de 14 años o más que poseen 3 años o menos de estudio

C.2 Asistencia a la escuela

(1) 1 o más personas en el hogar con edad entre 6 y 18 años que no asisten a la escuela

(0) No hay personas en el hogar con edad entre 6 y 18 años que no asisten a la escuela

D. Mercado de trabajo

D.1 Trabajo infantil

(1) 1 o más personas en el hogar con edad entre 5 y 17 años ocupadas

(0) No hay

D.2 Adultos sin trabajo

(1) 1 o más personas en el hogar clasificadas como: desocupadas con 18 años o más; inactivas que no asistían a la escuela y no recibían ningún tipo de ingreso; y personas ocupadas con edad inferior a 18 años

(0) No hay

Fuente: elaboración propia.

IPM: índice de privación multidimensional.

grado de protección de las viviendas de la intemperie, respectivamente. El número de personas que duermen en habitaciones utilizadas como dormitorios (indicador denominado "hogares en situación de hacinamiento") es otra variable importante para medir la capacidad de las personas en cuanto a su salud y su autoestima.

La inclusión del factor salud se debe a que el estado físico y psíquico de las personas constituye un aspecto fundamental en el desarrollo de su habilidad para ejercer muchas capacidades y tener libertad de elección en varios funcionamientos. Al respecto, se tienen en cuenta dos indicadores: la autoevaluación de la salud y el número de hijos nacidos que murieron, independientemente del sexo. El primer indicador sintetiza la percepción de las personas en lo que concierne a su propio estado de salud. Las respuestas "muy buena", "buena" y "regular" se agruparon en una sola categoría (saludables) y las respuestas "muy mala" y "mala" en otra (no saludables). 
De acuerdo con Noronha (2005), no hay consenso en la literatura sobre el mejor criterio para clasificar la evaluación del propio estado de salud y, en ese sentido, se optó por esta definición restrictiva de privación. El indicador hijos nacidos vivos que fallecieron es una medida indirecta de la muerte prematura y las normas generales de salud.

La presencia de analfabetos funcionales en el hogar y la asistencia escolar de los niños y adolescentes son los indicadores de la dimensión del índice relativa a la educación. Además de ser importante en sí misma, la educación es un instrumento de capacitación de los individuos en el sentido utilizado en el enfoque de las capacidades, pues facilita el ejercicio de la condición de agente en una serie de funcionamientos como gozar de buena salud, tener un trabajo digno, ejercer la ciudadanía, apreciar obras de arte y eventos culturales, entre otros.

La cuarta y última dimensión se describe mediante el trabajo infantil y la presencia de adultos sin trabajo en el hogar. La inserción en el mercado laboral, en edad compatible con el desarrollo humano, es un requisito decisivo para evitar la privación y alcanzar buena parte de los funcionamientos.

En las cuatro secciones siguientes se presentan los resultados del IPM. Cada una de ellas tiene un objetivo específico, con miras a presentar una visión integral de la privación en el Brasil urbano desde una perspectiva multidimensional. En la próxima sección se ofrece una visión general del índice.

\section{III}

\section{Resultados generales del índice de privación multidimensional (IPM)}

En esta sección se presentan los resultados generales del IPM con respecto al Brasil urbano. El análisis comprende los años 2003 y 2008, con el objetivo de medir los aspectos dinámicos de la privación reciente. Como se describió anteriormente, los hogares pueden clasificarse como no privados o registrar privación en por lo menos uno de los 13 indicadores. El número de personas que sufren privación por número de indicadores se detalla en el cuadro 3 .

En primer lugar se destaca que, si bien la población urbana brasileña aumentó de 132 millones a 152 millones de habitantes en el período de referencia, el número absoluto de personas en situación de privación, según un enfoque multidimensional, aumentó solo de 91 millones

CUADRO 3

Distribución de personas en situación de privación por número de indicadores

\begin{tabular}{|c|c|c|c|c|c|c|}
\hline \multirow{2}{*}{ Privación por número de indicadores } & \multicolumn{2}{|c|}{$\begin{array}{l}\text { Número de personas } \\
\quad(\text { en miles })\end{array}$} & \multicolumn{2}{|c|}{ Porcentaje } & \multicolumn{2}{|c|}{$\begin{array}{c}\text { Porcentaje de personas } \\
\text { con privación }\end{array}$} \\
\hline & 2003 & 2008 & 2003 & 2008 & 2003 & 2008 \\
\hline 0 & 41104 & 57489 & 31,1 & 38,0 & - & - \\
\hline 1 & 46363 & 51021 & 35,0 & 33,6 & 50,9 & 54,2 \\
\hline 2 & 27330 & 27232 & 20,7 & 18,0 & 30,0 & 28,9 \\
\hline 3 & 11866 & 10992 & 9,0 & 7,2 & 13,0 & 11,7 \\
\hline 4 & 4085 & 3671 & 3,1 & 2,4 & 4,5 & 3,9 \\
\hline 5 & 1173 & 1028 & 0,9 & 0,7 & 1,3 & 1,1 \\
\hline 6 & 244 & 197 & 0,2 & 0,1 & 0,3 & 0,2 \\
\hline 7 & 23 & 21 & 0,0 & 0,0 & 0,0 & 0,0 \\
\hline 8 & 11 & 6 & 0,0 & 0,0 & 0,0 & 0,0 \\
\hline 9 & 0 & 0 & 0,0 & 0,0 & 0,0 & 0,0 \\
\hline 10 o más & 0 & 0 & 0,0 & 0,0 & 0,0 & 0,0 \\
\hline Total en privación & 91096 & 94168 & 68,9 & 62,1 & 100,0 & 100,0 \\
\hline Total de la población & 132200 & 151657 & 100,0 & 100,0 & - & - \\
\hline
\end{tabular}

Fuente: elaboración propia sobre la base de Instituto Brasileño de Geografía y Estadística (IBGE), Encuesta Nacional de Hogares de 2003 y 2008 . 
a 94 millones. Esa cifra representa una reducción relativa del $68,9 \%$ al $62,1 \%$ de la población urbana. Así, en solo cinco años, la proporción de personas que no se hallaban en situación de privación se acrecentó en forma considerable, del 31,8\% al 37,9\%, como consecuencia de los recientes avances socioeconómicos en el país.

Con respecto a las personas en situación de privación por número de indicadores, cabe destacar que, como se puede apreciar en las dos últimas columnas del cuadro 3 , los hogares con privación respecto de un solo indicador representan el 50,9\% de las personas que vivían en hogares con privación en 2003 y el 54,2\% en 2008. La privación con respecto a seis o más indicadores es poco significativa y se vuelve nula con relación a 10 o más indicadores.

Asimismo, cabe notar el incremento relativo de la proporción de personas que residen en hogares con privación respecto de un solo indicador y la reducción en todas las demás categorías. Este resultado evidencia que la privación multidimensional en el Brasil disminuyó entre 2003 y 2008 debido a dos tendencias distintas: el número relativo de individuos en hogares con privación se redujo y, entre aquellos que ya se encontraban en situación de privación, creció la proporción con respecto a un único indicador.
En el cuadro 4 se muestran los resultados del IPM, según se describe en la sección metodológica (sección II), y se clasifica a los individuos pertenecientes a hogares con privación en por lo menos uno de los 13 indicadores como pobres. La proporción de personas que sufren privación $(\mathrm{H})$ de la población urbana del Brasil con por lo menos un indicador de privación es del 68,9\% en 2003 y el $61,7 \%$ en 2008. Si se tiene en cuenta la privación en "por lo menos dos indicadores", la proporción se reduce al $33,8 \%$ y el $28,0 \%$, respectivamente.

La intensidad media de las privaciones (A), calculada por la media ponderada de la privación multidimensional entre los pobres para cada categoría de los indicadores de privación, se presenta en la tercera y cuarta columnas. Se puede apreciar que el valor aumenta con la inclusión de un indicador más en la privación. Esto ocurre porque las personas que sufren privación en muchos aspectos son precisamente las que padecen esa condición con mayor intensidad. En las dos últimas columnas se muestra el IPM resultante del producto de $\mathrm{H}$ y A, en 2003 y 2008. Conforme esa medida, la privación multidimensional en el Brasil sería del $0,718 \%$ y del $0,584 \%$, respectivamente, para personas residentes en hogares con privación en por lo menos un indicador.

CUADRO 4

Brasil: índice de privación multidimensional (IPM), 2003 y 2008

\begin{tabular}{|c|c|c|c|c|c|c|}
\hline \multirow{2}{*}{$\begin{array}{l}\text { Número de indicadores de privación } \\
\text { (como mínimo) }\end{array}$} & \multicolumn{2}{|c|}{$\begin{array}{c}\mathrm{H} \\
\text { (en porcentajes) }\end{array}$} & \multicolumn{2}{|c|}{ A } & \multicolumn{2}{|c|}{ IPM } \\
\hline & 2003 & 2008 & 2003 & 2008 & 2003 & 2008 \\
\hline 1 & 68,9 & 61,7 & 0,010419 & 0,009453 & 0,718 & 0,584 \\
\hline 2 & 33,8 & 28,0 & 0,014732 & 0,014405 & 0,499 & 0,404 \\
\hline 3 & 13,2 & 10,2 & 0,019342 & 0,019978 & 0,255 & 0,203 \\
\hline 5 & 1,1 & 0,8 & 0,029085 & 0,030654 & 0,032 & 0,023 \\
\hline 6 & 0,2 & 0,1 & 0,034284 & 0,036870 & 0,007 & 0,005 \\
\hline 7 & 0,0 & 0,0 & 0,039192 & 0,045191 & 0,001 & 0,001 \\
\hline 8 & 0,0 & 0,0 & 0,043282 & 0,050437 & 0,000 & 0,000 \\
\hline 9 & 0,0 & 0,0 & 0,052198 & & 0,000 & \\
\hline 10 o más & 0,0 & 0,0 & & & & \\
\hline
\end{tabular}

Fuente: elaboración propia sobre la base de Instituto Brasileño de Geografía y Estadística (IBGE), Encuesta Nacional de Hogares de 2003 y 2008. 


\section{IV}

\section{Análisis del índice multidimensional por indicador}

En esta sección se examinan con más detalle los indicadores de privación utilizados para la construcción del IPM. Solo se presentan los resultados correspondientes al año 2008 debido a su similitud con los resultados relativos a 2003.

En el cuadro 5 se expone la distribución de la privación relativa a cada uno de los 13 indicadores, incluidas las personas que registran privación solo en un indicador específico - es decir, que sufren privaciones unidimensionales - y también aquellas que sufren privación en dos o más indicadores desde una perspectiva multidimensional. En el panel superior del cuadro 5 se presentan los resultados totales relativos a cada indicador, o sea, el número de personas con respecto a las cuales

CUADRO 5

Distribución de las personas por tipo de privación

Tipo de privación

Número de personas que sufren privación

(en miles)
Proporción de la población urbana del Brasil (en porcentajes)

\begin{tabular}{lrr}
\hline \multicolumn{2}{c}{ Población total por privación } & \\
\hline Alcantarillado & 47884 & 31,6 \\
Hijos nacidos que murieron & 24814 & 16,4 \\
Destino de los residuos domiciliarios & 14683 & 9,7 \\
Asistencia escolar & 12605 & 8,3 \\
Trabajo infantil & 11484 & 7,6 \\
Hogares en situación de hacinamiento & 10200 & 6,7 \\
Activos & 8389 & 5,5 \\
Agua & 8246 & 5,4 \\
Analfabetismo funcional & 7742 & 5,1 \\
Autoevaluación de la salud & 5373 & 3,5 \\
Adultos sin trabajo & 5256 & 3,5 \\
Tipo de combustible utilizado para cocinar & 2096 & 1,4 \\
Material de las paredes de la vivienda & 944 & 0,6 \\
\hline
\end{tabular}

Combinaciones de privación más numerosas

\begin{tabular}{lrr}
\hline Alcantarillado & 19249 & 12,1 \\
Hijos nacidos que murieron & 9008 & 5,6 \\
Destino de los residuos domiciliarios & 5066 & 3,2 \\
Alcantarillado / Hijos nacidos que murieron & 4286 & 2,8 \\
Asistencia escolar & 3592 & 2,3 \\
Hogares en situación de hacinamiento & 3295 & 2,1 \\
Trabajo infantil & 3192 & 2,0 \\
Activos & 2651 & 1,7 \\
Alcantarillado /Agua & 2589 & 1,7 \\
Alcantarillado / Destino de los residuos domiciliarios & 2317 & 1,5 \\
Alcantarillado / Asistencia escolar & 1733 & 1,1 \\
Alcantarillado / Trabajo infantil & 1662 & 1,1 \\
Alcantarillado / Hogares en situación de hacinamiento & 1578 & 1,0 \\
\hline & Otros para solo un indicador & \\
\hline Autoevaluación de la salud & & 0,9 \\
Agua & 1478 & 0,7 \\
Analfabetismo funcional & 1171 & 0,7 \\
Adultos sin trabajo & 1107 & 0,6 \\
Tipo de combustible utilizado para cocinar & 883 & 0,1 \\
Material de las paredes de la vivienda & 235 & 0,1
\end{tabular}

Fuente: elaboración propia sobre la base de Instituto Brasileño de Geografía y Estadística (IBGE), Encuesta Nacional de Hogares de 2008. 
el valor del indicador específico asume un valor igual a 1, tanto unidimensional como multidimensionalmente.

El indicador de privación más significativo desde el punto de vista numérico se refiere al servicio sanitario o sistema de disposición de las aguas residuales (alcantarillado), pues más de 47 millones de brasileños, que representan el $31,6 \%$ de la población urbana, registran carencias en este aspecto. En segundo lugar se encuentra el indicador "hijos nacidos que murieron", con más de 24 millones de brasileños en hogares con esa privación. El tercer indicador más común según el número de personas afectadas es el "destino de los residuos domiciliarios", con cifras superiores a 14 millones, seguido por "asistencia escolar", "trabajo infantil" y "hogares en situación de hacinamiento", que corresponden a más de 10 millones de ciudadanos cada uno. Con respecto a los demás indicadores, los números varían entre poco más de 8 millones de personas en situación de privación en el caso de los activos y menos de 1 millón de personas con respecto al material de las paredes de la vivienda.

Las cifras mencionadas se refieren al total de privación relativo a cada indicador. En el resto del cuadro 5 aparece un examen más detallado. Por ejemplo, muchas de las más de 47 millones de personas que carecen de alcantarillado en la vivienda sufren privación solo con respecto a este indicador, mientras que otras también resultan afectadas en otros indicadores. En otras palabras, en tanto que en algunos hogares se observan privaciones en un único indicador, en otros se registran con respecto a dos o más indicadores (Ferreira y Lugo, 2012). En el cuadro 5 se presentan las combinaciones específicas de privación más significativas numéricamente, pues alcanzan a más del 1\% de los hogares brasileños. Cabe señalar que el número de combinaciones es enorme, porque mientras la privación en un único indicador (o sea, el número de indicadores) supone 13 perfiles, la privación en dos indicadores cualesquiera (es decir, el número de posibilidades de que se agrupen dos indicadores diferentes de los 13 seleccionados) determina 72 perfiles; la privación en tres indicadores cualesquiera determina la creación de 286 perfiles, y así sucesivamente.

La combinación específica más numerosa corresponde al "alcantarillado", perfil de privación unidimensional que comprende a más de 19 millones de brasileños (sufren privación solo con respecto a ese indicador). A continuación aparecen los perfiles también unidimensionales ligados a los indicadores "hijos nacidos que murieron" y "destino de los residuos domiciliarios", con cifras superiores a los 5 millones de personas afectadas. En el cuarto tipo de perfil más frecuente se combinan dos indicadores: "alcantarillado" e "hijos nacidos que murieron", y representa la esfera de privación multidimensional más numerosa, con más de 4 millones de personas. En seguida figuran cuatro perfiles unidimensionales de privación: "asistencia escolar", "hogares en situación de hacinamiento", "trabajo infantil" y "activos" en el hogar, cada uno de los cuales abarca a más del 1,5\% de la población brasileña urbana. Les siguen otros cinco indicadores de privación bidimensional, que no incluyen el alcantarillado. Cabe señalar que ninguno de los perfiles bidimensionales sin el indicador alcantarillado es numeroso y que lo mismo ocurre con los perfiles con tres o más indicadores de privación.

En la parte inferior del cuadro 5 se aprecia que los otros seis perfiles unidimensionales de privación son menos significativos numéricamente, ya que los porcentajes varían entre un $0,1 \%$ y un $0,9 \%$ de la población urbana brasileña.

De acuerdo con el debate presentado en Ferreira y Lugo (2012), siempre que sea posible se debe examinar la privación multidimensional desde una perspectiva combinatoria con las distribuciones conjuntas. En el cuadro 6 se muestran los cinco perfiles de privación más numerosos con respecto a cada uno de los 13 indicadores. Por ejemplo, en el caso de las personas con privación en cuanto al servicio sanitario o sistema de disposición de las aguas residuales (alcantarillado), el perfil más numeroso era el unidimensional. A continuación figura la combinación bidimensional del indicador "alcantarillado" con el indicador "hijos nacidos que murieron", seguida por tres perfiles también bidimensionales formados por el indicador "alcantarillado", combinado respectivamente con los indicadores "agua", "recolección de los residuos domiciliarios" y "asistencia escolar". Estos resultados sugieren que el mejoramiento de las condiciones habitacionales relativas al alcantarillado puede tener consecuencias directas e indirectas en los niveles de privación en el Brasil urbano. En otras palabras, el incremento del acceso al alcantarillado tendría un efecto directo, debido a la disminución de la privación respecto de este indicador, y posiblemente también indirecto, pues podría influir en la privación ligada a los hijos nacidos que murieron, el agua, la recolección de los residuos domiciliarios o la asistencia escolar, entre otros factores.

Es posible realizar análisis similares con respecto a los demás indicadores. Por ejemplo, el perfil unidimensional del indicador "hijos nacidos que murieron" también resultó ser el más numeroso, seguido por cuatro combinaciones bidimensionales con los indicadores "alcantarillado", "destino de los residuos domiciliarios", "asistencia escolar" y "hogares en situación de hacinamiento", respectivamente. 
CUADRO 6

Distribución de los tipos de privación en por lo menos un indicador

\begin{tabular}{|c|c|c|c|c|c|}
\hline \multirow{2}{*}{ Indicador } & \multicolumn{5}{|c|}{ Más numerosos (con) } \\
\hline & Primero & Segundo & Tercero & Cuarto & Quinto \\
\hline Alcantarillado & - & $\begin{array}{l}\text { Hijos nacidos que } \\
\text { murieron }\end{array}$ & Agua & $\begin{array}{c}\text { Destino de } \\
\text { los residuos } \\
\text { domiciliarios }\end{array}$ & Asistencia escolar \\
\hline $\begin{array}{l}\text { Hijos nacidos que } \\
\text { murieron }\end{array}$ & - & Alcantarillado & $\begin{array}{c}\text { Destino de } \\
\text { los residuos } \\
\text { domiciliarios }\end{array}$ & Asistencia escolar & $\begin{array}{c}\text { Hogares en } \\
\text { situación de } \\
\text { hacinamiento }\end{array}$ \\
\hline $\begin{array}{l}\text { Destino de los residuos } \\
\text { domiciliarios }\end{array}$ & - & Alcantarillado & $\begin{array}{l}\text { Hijos nacidos que } \\
\text { murieron }\end{array}$ & $\begin{array}{l}\text { Hijos nacidos } \\
\text { que murieron / } \\
\text { Hogares en } \\
\text { situación de } \\
\text { hacinamiento }\end{array}$ & $\begin{array}{l}\text { Alcantarillado / } \\
\text { Agua }\end{array}$ \\
\hline Asistencia escolar & - & Alcantarillado & Trabajo infantil & $\begin{array}{l}\text { Hijos nacidos que } \\
\text { murieron }\end{array}$ & $\begin{array}{l}\text { Hijos nacidos } \\
\text { que murieron / } \\
\text { Alcantarillado }\end{array}$ \\
\hline $\begin{array}{l}\text { Hogares en situación de } \\
\text { hacinamiento }\end{array}$ & - & Alcantarillado & $\begin{array}{l}\text { Hijos nacidos que } \\
\text { murieron }\end{array}$ & Asistencia escolar & $\begin{array}{c}\text { Alcantarillado / } \\
\text { Hijos nacidos que } \\
\text { murieron }\end{array}$ \\
\hline Trabajo infantil & - & Alcantarillado & Asistencia escolar & $\begin{array}{c}\text { Asistencia } \\
\text { escolar / } \\
\text { Alcantarillado }\end{array}$ & $\begin{array}{l}\text { Hijos nacidos que } \\
\text { murieron }\end{array}$ \\
\hline Activos & - & $\begin{array}{l}\text { Analfabetismo } \\
\text { funcional }\end{array}$ & Alcantarillado & $\begin{array}{l}\text { Analfabetismo } \\
\text { funcional / } \\
\text { Adultos sin } \\
\text { trabajo }\end{array}$ & $\begin{array}{l}\text { Alcantarillado / } \\
\text { Analfabetismo } \\
\text { funcional }\end{array}$ \\
\hline $\begin{array}{l}\text { Autoevaluación de } \\
\text { la salud }\end{array}$ & - & Alcantarillado & $\begin{array}{l}\text { Hijos nacidos que } \\
\text { murieron }\end{array}$ & $\begin{array}{c}\text { Alcantarillado / } \\
\text { Hijos nacidos que } \\
\text { murieron }\end{array}$ & $\begin{array}{c}\text { Destino de } \\
\text { los residuos } \\
\text { domiciliarios }\end{array}$ \\
\hline Agua & Alcantarillado & Ninguno & $\begin{array}{l}\text { Alcantarillado / } \\
\text { Hijos nacidos que } \\
\text { murieron }\end{array}$ & $\begin{array}{l}\text { Alcantarillado / } \\
\text { Destino de } \\
\text { los residuos } \\
\text { domiciliarios }\end{array}$ & $\begin{array}{l}\text { Alcantarillado / } \\
\text { Trabajo infantil }\end{array}$ \\
\hline Analfabetismo funcional & - & Activos & Alcantarillado & $\begin{array}{l}\text { Hijos nacidos que } \\
\text { murieron }\end{array}$ & $\begin{array}{l}\text { Alcantarillado / } \\
\text { Activos }\end{array}$ \\
\hline Adultos sin trabajo & - & $\begin{array}{c}\text { Activos/ } \\
\text { Analfabetismo } \\
\text { funcional }\end{array}$ & Activos & $\begin{array}{l}\text { Analfabetismo } \\
\text { funcional }\end{array}$ & $\begin{array}{l}\text { Hijos nacidos que } \\
\text { murieron }\end{array}$ \\
\hline $\begin{array}{l}\text { Tipo de combustible } \\
\text { utilizado para cocinar }\end{array}$ & Alcantarillado & - & $\begin{array}{l}\text { Alcantarillado / } \\
\text { Hijos nacidos que } \\
\text { murieron }\end{array}$ & $\begin{array}{l}\text { Hijos nacidos que } \\
\text { murieron }\end{array}$ & $\begin{array}{l}\text { Alcantarillado / } \\
\text { Destino de } \\
\text { los residuos } \\
\text { domiciliarios } \\
\end{array}$ \\
\hline Material de las paredes & Alcantarillado & - & $\begin{array}{l}\text { Alcantarillado / } \\
\text { Hijos nacidos que } \\
\text { murieron }\end{array}$ & $\begin{array}{l}\text { Alcantarillado / } \\
\text { Destino de } \\
\text { los residuos } \\
\text { domiciliarios }\end{array}$ & $\begin{array}{c}\text { Hogares en } \\
\text { situación de } \\
\text { hacinamiento }\end{array}$ \\
\hline
\end{tabular}

Fuente: elaboración propia sobre la base de Instituto Brasileño de Geografía y Estadística (IBGE), Encuesta Nacional de Hogares de 2008.

Cabe destacar que en la mayoría de los casos el perfil más numeroso corresponde al del propio indicador considerado individualmente. Los indicadores relativos al tipo de agua, el combustible utilizado para cocinar y el material de las paredes de la vivienda constituyen excepciones, pues los perfiles más numerosos son los de privación bidimensional combinada con el indicador "alcantarillado". Se verificó también que existen perfiles tridimensionales relativamente numerosos, entre ellos los que combinan "destino de los residuos domiciliarios", "hijos nacidos que murieron" y "hogares en situación de hacinamiento"; o "asistencia escolar", "hijos 
nacidos que murieron" y "alcantarillado"; u "hogares en situación de hacinamiento", "alcantarillado" e "hijos nacidos que murieron", y así sucesivamente. Esto sugiere la existencia de sinergias negativas entre los indicadores y pone en evidencia diferentes realidades de privación múltiple.

No obstante, se observa que los niveles absolutos de privación de cada indicador, detallados en la parte superior del cuadro 5, influyen en esos resultados. La privación relativa al alcantarillado coincide en muchos hogares con la privación en otro indicador, porque el primero es el más numeroso de todos. Los resultados que no revelan una frecuencia absoluta, es decir, los indicadores cuya probabilidad de aparecer combinados con otros es mayor que la de figurar en forma aislada, se destacan en negrita en el cuadro 6. Por ejemplo, a pesar de que la privación de los hogares con respecto al agua no es significativa numéricamente, el indicador de privación bidimensional "alcantarillado-agua" sí lo es. Conviene subrayar, asimismo, la relación entre el trabajo infantil y la asistencia escolar, pues los valores absolutos de la combinación de ambos indicadores también son más significativos que los de cada uno de ellos por separado. Si bien no son significativos desde la perspectiva de un solo indicador, los aspectos relativos a la presencia de activos, analfabetos funcionales y adultos sin trabajo en el hogar también están relacionados.

Los resultados presentados en el cuadro 6 dejan ver que algunos indicadores específicos de la privación tienden a ser más significativos al examinarse conjuntamente en dos o tres dimensiones que en forma independiente. Para entender mejor esas relaciones se realizó un análisis factorial (véase el cuadro 7), con el objetivo de verificar si realmente existía una correlación positiva entre los indicadores citados, por ejemplo, si los hogares con carencias en el indicador "alcantarillado" realmente tendían a presentar privación también con respecto al indicador "agua".

El análisis factorial de los perfiles destacados en el cuadro 6 confirmó algunos de los resultados presentados, puesto que se obtuvieron distintos grupos de indicadores. Para facilitar la interpretación, los indicadores "alcantarillado" y "agua" se agruparon en una única dimensión denominada "características urbanas" y los indicadores "trabajo infantil" y "asistencia escolar" en una dimensión denominada "niños". Se formó también un grupo con tres indicadores ("adultos sin trabajo", "analfabetismo funcional" y "activos") denominado "adultos sin calificación de bajos ingresos". El análisis factorial reveló la existencia de otro grupo, que no se observó en el cuadro 6 debido al bajo número de observaciones, formado por los indicadores "material predominante en la construcción de las paredes externas de la vivienda" y "tipo de combustible utilizado para cocinar" y que refleja un estatus socioeconómico bajo.

Los indicadores "número de hijos nacidos que murieron", "destino de los residuos domiciliarios", "autoevaluación de la salud" y "hogares en situación de hacinamiento" son relativamente independientes de los demás y no se agruparon con ningún otro. Estos resultados sugieren que las políticas para reducir la privación multidimensional podrían concentrarse en las sinergias existentes entre los indicadores. Por ejemplo, la calificación de los adultos con baja escolaridad probablemente tendría efectos positivos en los indicadores "analfabetismo funcional" y "activos".

CUADRO 7

Grupos de dimensiones clasificados por el análisis factorial

1. Características urbanas

Servicio sanitario o sistema de disposición de las aguas residuales y proveniencia del agua por cañería utilizada en la vivienda

2. Niños

Trabajo infantil y asistencia escolar

3. Adultos no calificados de bajos ingresos

Adultos sin trabajo, analfabetos funcionales y activos en el hogar

4. Estatus socioeconómico muy bajo similar a un ambiente rural

Material predominante en la construcción de las paredes externas de la vivienda y tipo de combustible utilizado para cocinar

5. Número de hijos nacidos que murieron

6. Destino de los residuos domiciliarios

7. Autoevaluación de la salud

8. Hogares en situación de hacinamiento

Fuente: elaboración propia. 


\section{V \\ Comparación de los índices monetario y multidimensional a nivel regional}

En esta sección se compara la privación multidimensional con la privación medida según la métrica monetaria en los estados brasileños. Para ello es necesario definir los hogares que sufren privación monetaria y no monetaria.

En primer lugar, se define a los pobres desde el punto de vista monetario. Los ingresos del hogar en 2003 -es decir, la suma de los ingresos individuales de todas las personas del hogar - se dividieron entre el número de miembros de la unidad, para obtener así los ingresos del hogar per cápita. Ese valor se dividió a su vez entre el valor del salario mínimo en septiembre de 2003 - fecha de referencia de la PNAD de ese año (240 reales)—, y una cifra definida a nivel nacional. Se obtuvieron así los ingresos del hogar per cápita en salarios mínimos de septiembre de 2003 de todos los hogares brasileños y se clasificó como pobres a los residentes en hogares en los que dichos ingresos eran inferiores al 50\% del salario mínimo. Cabe señalar que ese valor fue adoptado por el gobierno para las políticas de programas sociales y se utiliza comúnmente en los estudios sobre la privación en el Brasil (véanse, por ejemplo, Banco Mundial, 2006; y un análisis detallado sobre la definición de la línea de privación en el Brasil en Rocha, 2003). Este mismo procedimiento se aplicó también con respecto al año 2008 y los valores se ajustaron según el índice de precios al consumidor (IPC) del Instituto Brasileño de Geografía y Estadística (IBGE) al comparar con septiembre de 2003.

Conforme la definición adoptada en este trabajo, el $27 \%$ y el $16,1 \%$ de los habitantes de las zonas urbanas del Brasil fueron considerados pobres en 2003 y 2008, respectivamente, porcentajes que suponen una reducción considerable de la privación monetaria. Con respecto a la privación multidimensional, se hizo una distinción entre los hogares sin privación o con privación en por lo menos uno de los 13 indicadores.

En los gráficos 1 y 2 se compara la privación monetaria con los resultados presentados en el cuadro 4 , relativos a las personas que registraron por lo menos un indicador de privación en los años 2003 y 2008. Debido a que las proporciones de pobres según la medida del ingreso y según el IPM se aproximan cuando el número de indicadores de privación es como mínimo dos, se definió ese número arbitrario como base para clasificar a los hogares. En consecuencia, aquellos que sufren

GRÁFICO 1

Brasil (áreas urbanas): proporción de personas en situación de privación según los índices monetario y multidimensional, 2003

(En porcentajes)

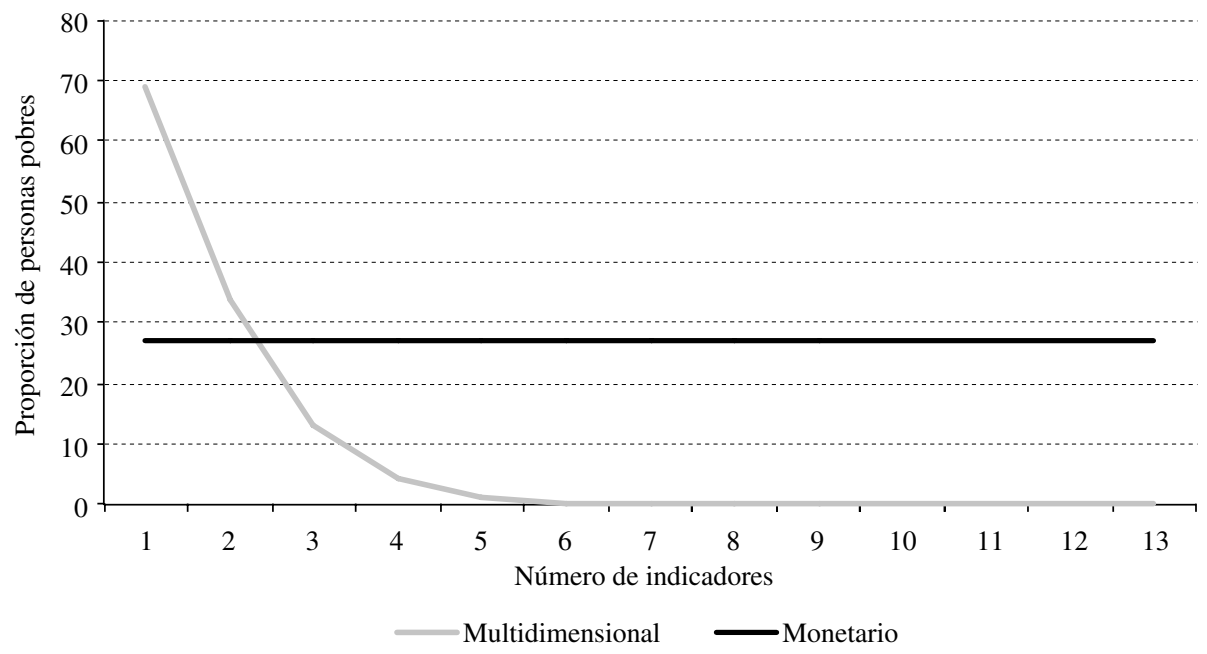

Fuente: elaboración propia sobre la base de Instituto Brasileño de Geografía y Estadística (IBGE), Encuesta Nacional de Hogares de 2003. 
GRÁFICO 2

Brasil (áreas urbanas): proporción de personas en situación de privación según los índices monetario y multidimensional, 2008

(En porcentajes)

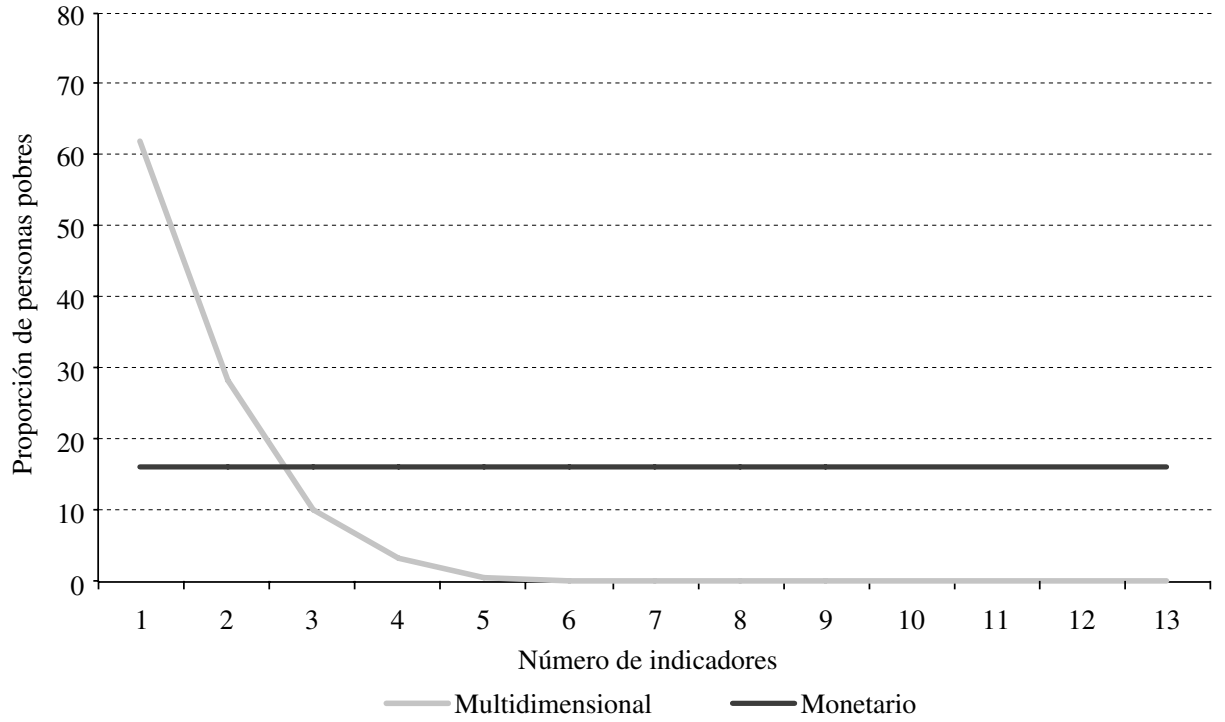

Fuente: elaboración propia sobre la base de Instituto Brasileño de Geografía y Estadística (IBGE), Encuesta Nacional de Hogares de 2008.

de privación con respecto a dos o más indicadores serán clasificados como pobres desde la perspectiva multidimensional.

En los mapas que se insertan a continuación se muestran las características de la privación monetaria y no monetaria en los estados brasileños. En los mapas 1 y 2 se aprecia la distribución de las personas en situación de privación según la medida de los ingresos en 2003 y 2008, respectivamente, mientras que el carácter multidimensional de la privación en los mismos años puede observarse en los mapas 3 y 4 .

De la lectura de los mapas 1 y 2 se concluye que los estados con mayor proporción de personas que sufren privación en los dos años, según la medida monetaria, fueron: Alagoas, Ceará, Maranhão, Paraíba, Pernambuco y Piauí, localizados en la región nordeste, mientras que los estados con una proporción inferior de personas que padecen privación se encuentran en las regiones sur y sudeste del Brasil: Paraná, Río de Janeiro, Rio Grande do Sul, Santa Catarina y São Paulo. En los dos períodos puede apreciarse un gradiente de privación de la región sur a las regiones norte y nordeste del Brasil. Asimismo, los mapas revelan una reducción de la proporción de personas que sufren privación en todos los estados.

En los mapas 3 y 4 se muestra la proporción de personas en hogares con privación en por lo menos dos indicadores. Los mayores valores relativos a la privación no monetaria -más del $70 \%$ en 2003 y del $50 \%$ en 2008- se registran en cuatro estados de las regiones norte y nordeste, a saber: Amapá, Pará, Piauí y Rondônia. Por otra parte, en el Distrito Federal, Minas Gerais y São Paulo se observan valores inferiores al $25 \%$ y el $20 \%$ en 2003 y 2008, respectivamente. En el área formada por las regiones norte, nordeste y centrooeste del Brasil se registran las cifras más altas. Se trata de una zona de colonización reciente, sobre todo las fronteras sur y este de la selva amazónica, donde aumenta cada vez más la producción de productos básicos agrícolas para exportación y las ciudades todavía no se han desarrollado completamente. Los principales componentes de la privación del área se relacionan con la urbanización (agua por cañería, alcantarillado y destino de los residuos domiciliarios, entre otros). Cabe destacar la marcada reducción de la privación multidimensional en la mayoría de los estados en el período de referencia.

En los mapas se refleja la desigualdad socioeconómica brasileña. Se observa que la privación, tanto medida monetariamente como de forma multidimensional, disminuyó de manera considerable en muchos estados, reducción que denota una notable evolución dinámica en los indicadores de privación monetarios y no monetarios. 
MAPA 1

\section{Brasil (áreas urbanas): proporción de personas en situación de privación} según los ingresos, 2003

(En porcentajes)

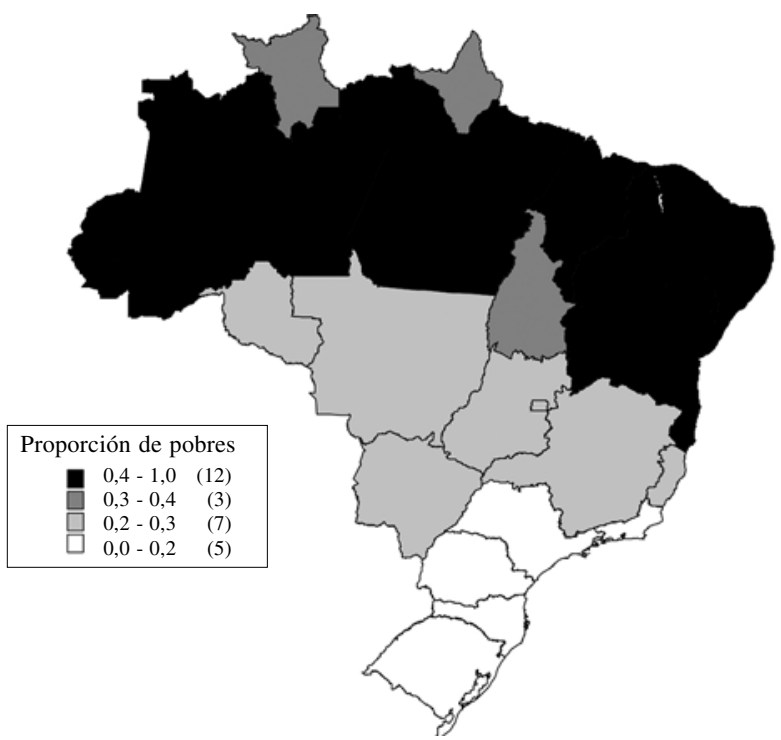

Fuente: elaboración propia sobre la base de Instituto Brasileño de Geografía y Estadística (IBGE), Encuesta Nacional de Hogares de 2003. Nota: números entre paréntesis indican el número de estados agrupados en un determinado rango de pobreza.

MAPA 2

Brasil (áreas urbanas): proporción de personas en situación de privación según los ingresos, 2008

(En porcentajes)

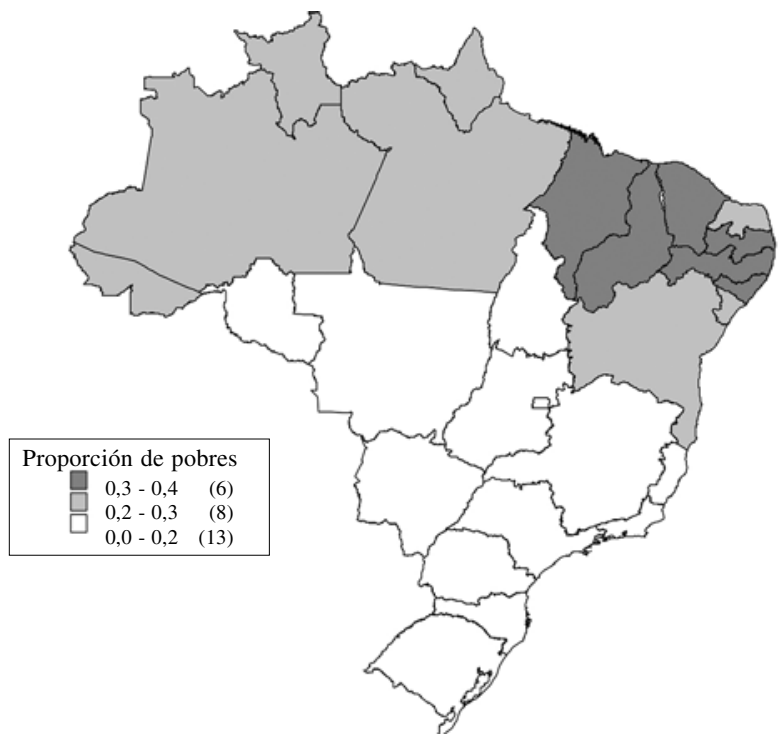

Fuente: elaboración propia sobre la base de Instituto Brasileño de Geografía y Estadística (IBGE), Encuesta Nacional de Hogares de 2008.

Nota: números entre paréntesis indican el número de estados agrupados en un determinado rango de pobreza. 
MAPA 3

Brasil (áreas urbanas): proporción de hogares en situación de privación en por lo menos dos indicadores, 2003

(En porcentajes)

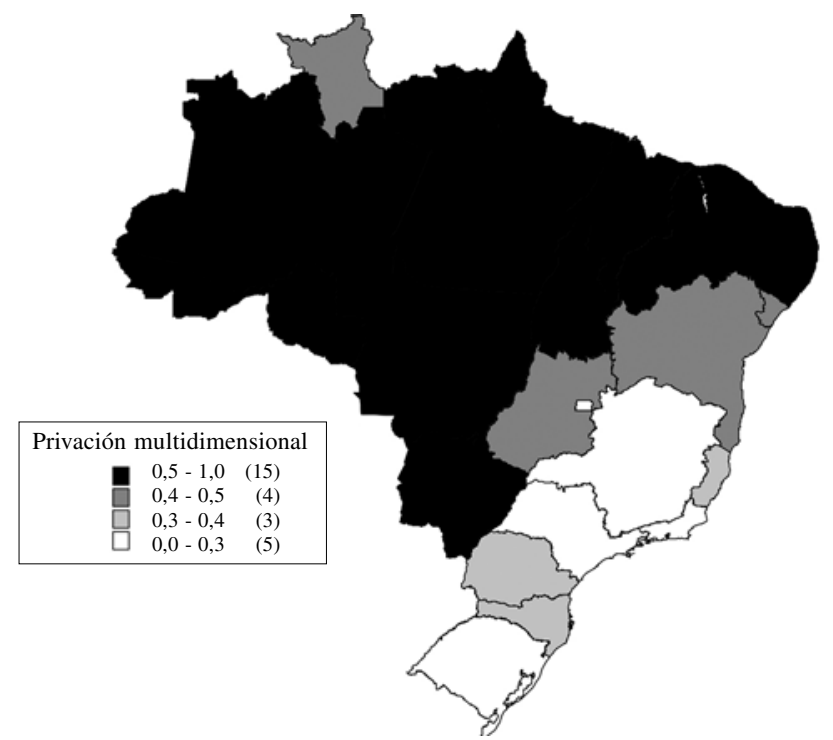

Fuente: elaboración propia sobre la base de Instituto Brasileño de Geografía y Estadística (IBGE), Encuesta Nacional de Hogares de 2003. Nota: números entre paréntesis indican el número de estados agrupados en un determinado rango de pobreza.

MAPA 4

Brasil (áreas urbanas): proporción de hogares en situación de privación en por lo menos dos indicadores, 2008

(En porcentajes)

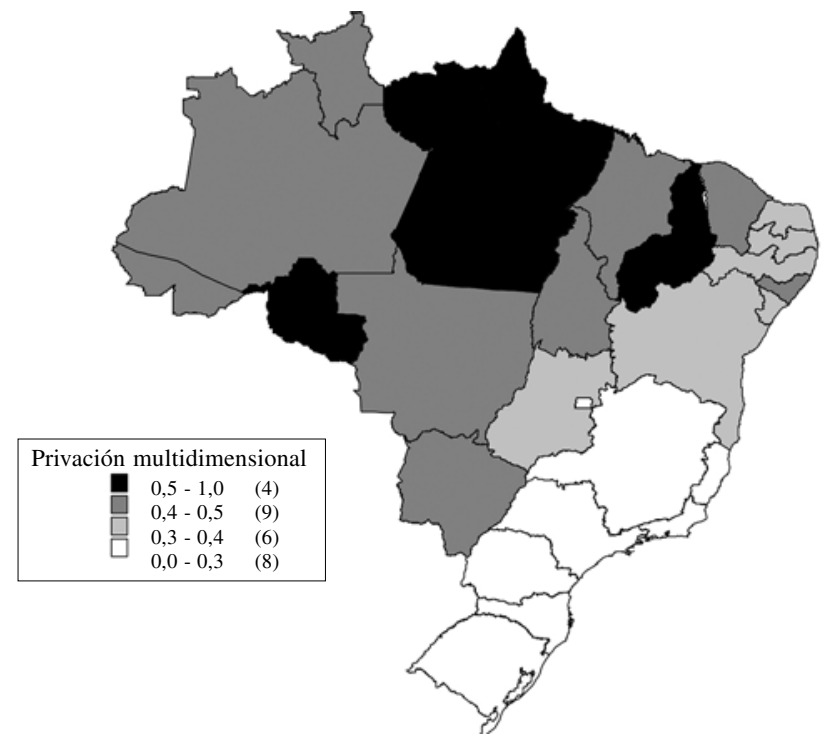

Fuente: elaboración propia sobre la base de Instituto Brasileño de Geografía y Estadística (IBGE), Encuesta Nacional de Hogares de 2008.

Nota: números entre paréntesis indican el número de estados agrupados en un determinado rango de pobreza. 
En el cuadro 8 se presenta la correlación entre esos indicadores de privación y, como se puede apreciar, todos ellos son positivos y significativos al 5\%. Al comparar un mismo indicador en dos años distintos, se observa una marcada correlación (ambas medidas por sobre el 95\%), mientras que los valores de la correlación entre el indicador monetario y el multidimensional son mucho más bajos (alrededor de 60\%). Además, la menor correlación de la privación multidimensional con el indicador basado en los ingresos en 2008 sugiere un aumento de las diferencias entre los dos tipos de medición.

En las últimas secciones se presentó una visión general del IPM, se analizaron algunas especificidades de cada dimensión de privación, sus regularidades y relaciones y se realizaron comparaciones con la privación monetaria. En líneas generales, los resultados revelaron una reducción de la privación multidimensional en el Brasil entre 2003 y 2008, que obedece a dos razones: la disminución del número relativo de personas en hogares con privación y, entre las personas que sufren carencias, el aumento de aquellas con registro en por lo menos un indicador. La privación monetaria también aminoró en el período de referencia.

Los resultados manifiestan la dinámica de la evolución de los indicadores. En la sección VI se presenta un enfoque más detallado de la dinámica de la privación y de la privación mediante el uso de cohortes sintéticas y modelos econométricos.

CUADRO 8

Correlación entre la privación monetaria medida según los ingresos y la privación multidimensional

\begin{tabular}{lccc}
\hline Indicadores & $\begin{array}{c}\text { Privación monetaria } \\
\text { en 2003 }\end{array}$ & $\begin{array}{c}\text { Privación monetaria } \\
\text { en 2008 }\end{array}$ & $\begin{array}{c}\text { Privación multidimensional } \\
\text { en 2003 }\end{array}$ \\
\hline Privación monetaria en 2008 & 0,98 & - & - \\
Privación multidimensional en 2003 & 0,69 & 0,62 & - \\
Privación multidimensional en 2008 & 0,59 & 0,52 & 0,96 \\
\hline
\end{tabular}

Fuente: elaboración propia sobre la base de Instituto Brasileño de Geografía y Estadística (IBGE), Encuesta Nacional de Hogares de 2003 y 2008 .

\section{VI}

\section{Análisis de la dinámica de privación por cohortes sintéticas}

En esta sección se analiza la dinámica de privación mediante el uso de cohortes sintéticas y modelos econométricos inspirados en el análisis edad-períodocohorte (Ben-Schlomo y Kuh, 2002; Reither, Hauser y Yang, 2009; Yang, 2007, 2008a y 2008b; Yang y Lee, 2009). Más específicamente, se procura responder a las siguientes preguntas: ¿ Son diferentes la distribución de la privación monetaria y de la privación multidimensional entre los distintos grupos de la población? ¿Se observa la misma tendencia temporal para esos grupos con respecto a dichos indicadores?

Debido a la ausencia de datos longitudinales se trabajó con cohortes sintéticas definidas por las características del jefe del hogar. Si bien las personas no son las mismas en los dos años analizados, el carácter representativo de la base de datos a nivel nacional determina que las cohortes sintéticas definen aproximadamente el mismo grupo de la población en los dos períodos y permite el análisis de los puntos relacionados con la dinámica temporal. En otras palabras, los datos individuales longitudinales se mimetizan con los datos de las cohortes sintéticas homogéneas y representativas.

La clasificación de las cohortes sintéticas se realizó por grupos homogéneos mediante el uso de cuatro variables: raza o color (blanco o negro), sexo (masculino o femenino), franja etaria (20-29, 30-39, 40-49, 50-59 y 60-69 años en 2003, y cinco años más en 2008) y nivel de escolaridad en años de educación formal (0, 1-3, 4-7, 8-10, 11, 12 años o más). Con estas categorías se crearon $2 \times 2 \times 5 \times 6=120$ cohortes sintéticas. Para que 
esos grupos fueran lo más homogéneos y representativos posibles se estableció un mínimo de 50 observaciones por grupo en cada año. De ese modo, las opciones se realizaron teniendo en cuenta un equilibrio entre homogeneidad y representatividad.

Asimismo, las características utilizadas en la definición de las cohortes sintéticas permiten seleccionar grupos muy similares en los dos años estudiados. Mientras que los cambios en el sexo de las personas son extremadamente raros, los cambios en la raza o color son más comunes, pues se trata de una variable definida por el declarante que puede cambiar de opinión en cuanto a su raza o color. La mayoría de los jefes de hogar tienen 20 o más años de edad y no presentan cambios en su nivel de educación formal, conforme con la categorización considerada. Si bien la migración internacional también puede alterar la composición de la población urbana, su volumen es pequeño en comparación con la población brasileña.

En todos los grupos homogéneos se determinó si el jefe de hogar pertenecía a un hogar considerado pobre según la medida monetaria y se lo clasificó conforme con el número de indicadores en que registraba privaciones. En el cuadro 9 se muestran los grupos de la población que presentaron mayor propensión a la privación monetaria o multidimensional. Se observan claramente algunas tendencias: la privación, según las dos definiciones, tiende a ser mayor en los hogares encabezados por personas negras en comparación con las blancas, así como en aquellos encabezados por mujeres. Los valores de los tres indicadores relativos a los hombres blancos jefes de hogar son inferiores a la media en los dos años considerados, mientras que en las demás categorías se registraron valores superiores a la media, que reflejan una mayor propensión a la privación monetaria y multidimensional. Se aprecia también una disminución de los dos tipos de indicadores de privación en 2008 en comparación con el año 2003.

De acuerdo con la medida monetaria, la proporción de hogares pobres es menor entre las cohortes de más edad en los dos años analizados. No hay una tendencia clara en cuanto a la privación en por lo menos un indicador, ya que en todas las cohortes se registran valores similares de alrededor del $70 \%$. Con respecto a la privación en por lo menos dos indicadores, se verifican valores similares para las cuatro cohortes más jóvenes en 2003 y para las tres primeras en 2008. Los mayores valores se registraron entre los integrantes de las cohortes de más edad y las diferencias aumentan en el período analizado.

CUADRO 9

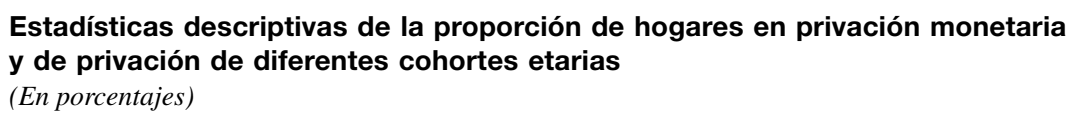

\begin{tabular}{|c|c|c|c|c|c|c|}
\hline \multirow{2}{*}{ Categorías } & \multicolumn{2}{|c|}{ Privación monetaria } & \multicolumn{2}{|c|}{$\begin{array}{l}\text { Privación como mínimo } \\
\text { en un indicador }\end{array}$} & \multicolumn{2}{|c|}{$\begin{array}{l}\text { Privación como mínimo } \\
\text { en dos indicadores }\end{array}$} \\
\hline & 2003 & 2008 & 2003 & 2008 & 2003 & 2008 \\
\hline Total & 28,0 & 16,3 & 71,0 & 66,8 & 36,9 & 34,4 \\
\hline Blancos & 17,8 & 9,4 & 67,4 & 63,3 & 33,2 & 31,2 \\
\hline Negros & 39,2 & 22,8 & 74,6 & 70,3 & 40,7 & 37,6 \\
\hline Hombres & 27,0 & 15,0 & 69,1 & 64,0 & 34,1 & 31,7 \\
\hline Mujeres & 31,2 & 18,9 & 72,8 & 69,6 & 39,7 & 37,1 \\
\hline \multicolumn{7}{|c|}{ Cohortes (edad en 2003) } \\
\hline $20-29$ & 38,4 & 23,8 & 71,1 & 67,5 & 36,4 & 32,3 \\
\hline $30-39$ & 33,7 & 19,4 & 71,1 & 66,6 & 35,9 & 31,7 \\
\hline $40-49$ & 26,2 & 13,6 & 71,4 & 64,9 & 36,2 & 32,3 \\
\hline $50-59$ & 21,4 & 10,9 & 69,7 & 65,9 & 35,6 & 34,6 \\
\hline $60-69$ & 18,4 & 6,5 & 71,7 & 69,1 & 40,5 & 41,1 \\
\hline \multicolumn{7}{|c|}{ Nivel de escolaridad (años) } \\
\hline 0 & 54,1 & 33,4 & 80,3 & 76,9 & 51,5 & 46,2 \\
\hline 1 a 3 & 42,9 & 27,9 & 79,7 & 79,8 & 51,6 & 47,1 \\
\hline 4 a 7 & 31,7 & 19,3 & 74,0 & 70,5 & 40,2 & 35,4 \\
\hline 8 a 10 & 22,1 & 15,0 & 65,8 & 64,7 & 29,4 & 28,7 \\
\hline 11 & 10,6 & 7,1 & 63,0 & 58,4 & 24,7 & 20,9 \\
\hline 12 y más & 3,2 & 2,4 & 63,7 & 55,0 & 21,1 & 15,6 \\
\hline
\end{tabular}

Fuente: elaboración propia sobre la base de Instituto Brasileño de Geografía y Estadística (IBGE), Encuesta Nacional de Hogares de 2003 y 2008. 
Los mayores niveles de escolaridad reducen la privación en los dos años analizados y en relación con todas las variables, pero sobre todo respecto de la privación monetaria. Se aprecia además que los valores de 2008 son menores que los de 2003, lo que indica la evolución positiva en los tres indicadores. No obstante, la evolución de la privación monetaria fue más acentuada que la de los demás indicadores.

En el cuadro 9 se presenta una visión general de los resultados. Para un análisis más profundo del efecto de las variables en la privación, se estimaron algunos modelos econométricos, teniendo en cuenta tres variables dependientes: privación monetaria, privación en por lo menos un indicador y privación en por lo menos dos indicadores. Las variables independientes son: una variable ficticia para el sexo ( 1 para masculino, 0 en caso contrario), una variable ficticia para la raza (1 para blanco, 0 para raza negra), variables ficticias para las cohortes (la más joven fue la categoría de referencia), cinco variables ficticias para los niveles de escolaridad (los menos instruidos como referencia) y una variable ficticia para el año ( 1 para 2008, 0 en caso contrario).

El análisis de las cohortes sintéticas se basó en cuatro modelos, estimados según el método de mínimos cuadrados ordinarios (MCO). El primero se refiere a los datos de 2003, el segundo a los datos de 2008, el tercero a los datos agregados (análisis similar a un panel con efectos aleatorios) y el cuarto a la variación en los valores en los años 2003 y 2008.

En el cuadro 10 se presentan los resultados. Se verifican algunas tendencias generales con respecto a los tres primeros modelos y a las tres variables dependientes. La privación monetaria y la privación respecto de otros indicadores son mayores en los hogares encabezados por personas de raza negra y por mujeres, incluso después del control mediante otras variables.

Históricamente, las personas de raza negra en el Brasil han estado sobrerrepresentadas en los estratos de menores ingresos y de mayor privación en la sociedad. Entre los factores que explican esa situación, y tal vez el más importante en la actualidad, está la brecha educativa entre blancos y negros. Las políticas aplicadas recientemente en el Brasil han casi universalizado la educación primaria, aumentado el acceso a la enseñanza media e incrementado el número de personas en la red de enseñanza privada, mientras que las políticas de acciones afirmativas han tenido el mismo efecto con respecto a los universitarios. Sin embargo, las personas con menores niveles de ingresos todavía deben lidiar con escuelas primarias de baja calidad y su menor nivel educativo les impide realizar un avance social real.
Al analizar la dinámica de estos resultados, los coeficientes fueron no significativos o de escasa magnitud. El coeficiente relativo a la raza fue positivo y significativo estadísticamente, tanto con respecto a la privación monetaria como a la privación en por lo menos dos indicadores, mientras que resultó no significativo en el modelo con privación como mínimo en un indicador. Esto sugiere que los hogares encabezados por personas de raza negra presentan una dinámica de privación monetaria similar a la de los demás, pero con un leve aumento en las diferencias entre los grupos raciales, en especial con relación a la privación multidimensional.

En el caso de los hogares encabezados por mujeres, los coeficientes negativos y significativos en los tres primeros modelos no constituyen una sorpresa. La ausencia de un cónyuge en la mayoría de esos hogares determina en parte los niveles más bajos de ingresos. Los hogares con parejas, muchos de los cuales cuentan a lo menos con dos fuentes de ingresos, tienden a estar encabezados por un hombre. Al comparar los hogares sin cónyuge, encabezados por hombres o mujeres, las diferencias son mucho menores. El último modelo, en el que se analiza la diferencia temporal en el indicador, reveló coeficientes negativos y significativos con respecto a la privación como mínimo en un indicador y no significativos en los demás casos. Pese a que estos resultados sugieren que la situación de los hogares encabezados por mujeres es peor con respecto a este indicador, en este caso se observó una ligera tendencia a la homogeneización de la privación no monetaria. Las políticas sociales recientemente implementadas en el Brasil, que son particularmente eficaces para este tipo de hogares, como la ampliación del acceso de los niños de seis años de edad a la escuela, podrían haber influido positivamente.

Cualquiera sea la variable dependiente considerada, se observa la misma tendencia con respecto al nivel educativo. A medida que el nivel de escolaridad desciende, se acrecienta la proporción de privación. Aunque estos resultados eran esperados, las tendencias temporales son diferentes. Como se puede apreciar en el último modelo, si bien las diferencias entre los niveles de escolaridad aminoraron la privación monetaria, en los hogares con mayor nivel educativo aumentó la propensión relativa a la privación. Este resultado puede explicarse en parte por el aumento general de los niveles educativos en el Brasil, la disminución de la desigualdad de ingresos debido a las políticas sociales de transferencia de ingresos (como el programa Bolsa Família y el Beneficio de Prestación Continuada), y por factores relacionados con el mercado de trabajo, entre otros. Por otra parte, el incremento de esas diferencias con respecto a la privación relativa a 
CUADRO 10

Análisis de los valores y las variaciones de los modelos econométricos estimados a partir de las cohortes sintéticas

\begin{tabular}{|c|c|c|c|c|c|}
\hline \multirow{2}{*}{ Variables } & & \multicolumn{3}{|c|}{ Valores } & \multirow{2}{*}{ Diferencia } \\
\hline & & 2003 & 2008 & Datos agregados & \\
\hline \multicolumn{6}{|c|}{ Privación monetaria } \\
\hline $\begin{array}{c}\text { Constante } \\
\text { Raza } \\
\text { Sexo }\end{array}$ & & $\begin{array}{r}0,80 \\
-0,11 \\
-0,05\end{array}$ & $\begin{array}{r}0,56 \\
-0,07 \\
-0,04\end{array}$ & $\begin{array}{r}0,74 \\
-0,09 \\
-0,04\end{array}$ & $\begin{array}{r}-0,24 \\
0,04 \\
\mathbf{0 , 0 1}\end{array}$ \\
\hline $\begin{array}{l}\text { Nivel de escolaridad } \\
\text { (años de educación formal) }\end{array}$ & $\begin{array}{l}1 \text { a } 3 \\
4 \text { a } 7 \\
8 \text { a } 10 \\
11 \\
12 \text { y más }\end{array}$ & $\begin{array}{l}-0,11 \\
-0,24 \\
-0,34 \\
-0,45 \\
-0,52\end{array}$ & $\begin{array}{l}-0,07 \\
-0,18 \\
-0,22 \\
-0,29 \\
-0,33\end{array}$ & $\begin{array}{l}-0,09 \\
-0,21 \\
-0,28 \\
-0,37 \\
-0,43\end{array}$ & $\begin{array}{l}0,04 \\
0,06 \\
0,12 \\
0,16 \\
0,19\end{array}$ \\
\hline Cohorte (edad en 2003) & $\begin{array}{l}30-39 \\
40-49 \\
50-59 \\
60-69\end{array}$ & $\begin{array}{l}-0,05 \\
-0,16 \\
-0,24 \\
-0,30\end{array}$ & $\begin{array}{l}-0,09 \\
-0,17 \\
-0,23 \\
-0,28\end{array}$ & $\begin{array}{l}-0,07 \\
-0,16 \\
-0,23 \\
-0,29\end{array}$ & $\begin{array}{r}-0,04 \\
\mathbf{- 0 , 0 2} \\
\mathbf{0 , 0 1} \\
\mathbf{0 , 0 2}\end{array}$ \\
\hline Año (2003) & & - & - & $-0,12$ & - \\
\hline
\end{tabular}

Año (2003) $\quad$ Privación como mínimo en un indicador

\begin{tabular}{|c|c|c|c|c|c|}
\hline & 90,9 & 89,4 & 88,1 & $-1,5$ \\
\hline \multicolumn{2}{|l|}{ Raza } & $-7,4$ & $-6,9$ & $-7,2$ & 0,5 \\
\hline \multicolumn{2}{|l|}{ Sexo } & $-3,1$ & $-4,8$ & $-3,9$ & $-1,8$ \\
\hline $\begin{array}{l}\text { Nivel de escolaridad } \\
\text { (años de educación formal) }\end{array}$ & $\begin{array}{l}1 \text { a } 3 \\
4 \text { a } 7 \\
8 \text { a } 10 \\
11 \\
12 \text { y más }\end{array}$ & $\begin{array}{r}-4,0 \\
-11,3 \\
-16,9 \\
-18,3 \\
-18,9\end{array}$ & $\begin{array}{r}-2,7 \\
-10,3 \\
-16,8 \\
-21,3 \\
-26,0\end{array}$ & $\begin{array}{r}-3,4 \\
-10,8 \\
-16,8 \\
-19,8 \\
-22,4\end{array}$ & $\begin{array}{r}\mathbf{1 , 4} \\
\mathbf{1 , 0} \\
\mathbf{- 0 , 1} \\
-3,0 \\
-7,1\end{array}$ \\
\hline Cohorte (edad en 2003) & $\begin{array}{l}30-39 \\
40-49 \\
50-59 \\
60-69\end{array}$ & $\begin{array}{r}-\mathbf{0 , 2} \\
\mathbf{0 , 1} \\
-2,1 \\
-0,1\end{array}$ & $\begin{array}{r}\mathbf{- 0 , 8} \\
-3,1 \\
-2,2 \\
\mathbf{0 , 8}\end{array}$ & $\begin{array}{r}\mathbf{- 0 , 5} \\
-1,5 \\
-2,2 \\
\mathbf{0 , 3}\end{array}$ & $\begin{array}{r}-\mathbf{0 , 6} \\
-3,1 \\
\mathbf{- 0 , 1} \\
\mathbf{0 , 9}\end{array}$ \\
\hline Año (2003) & & - & - & $-4,0$ & - \\
\hline
\end{tabular}

Fuente: elaboración propia sobre la base de Instituto Brasileño de Geografía y Estadística (IBGE), Encuesta Nacional de Hogares de 2003 y 2008.

Nota: los coeficientes en negrita no son significativos. 
por lo menos un indicador, especialmente en los hogares cuyos jefes iniciaron la enseñanza superior, sugiere que las personas más escolarizadas tienen más éxito en la superación de la privación no monetaria.

El resultado relativo a las cohortes varía según la variable dependiente empleada. En el caso de la privación medida por los ingresos, la tendencia es clara: la propensión a la privación es menor entre las cohortes de más edad. Este resultado refleja una peor inserción de los jóvenes en el mercado de trabajo, que puede estar ligada a tendencias recientes como las altas tasas de evasión escolar y el abuso de drogas, relacionado con las dimensiones de la epidemia de crack.
Muchos coeficientes referidos a la privación en por lo menos un indicador fueron no significativos, definiendo un perfil ligeramente diferente de la privación monetaria, ya sea en cuanto a los valores como a las diferencias. Pese a que la edad repercute en el tipo de privación, no se aprecian grandes disimilitudes respecto de las personas con privación no monetaria. Por otra parte, al analizar la privación en por lo menos dos indicadores, se registran valores más elevados entre las cohortes de edad más avanzada, que sugieren una mayor incidencia de la privación multidimensional entre los individuos de mayor edad, así como un aumento de las diferencias en el período referido.

\section{VII}

\section{Comentarios finales y conclusiones}

Utilizando el enfoque de las capacidades como referencial teórico, en este trabajo se presentó un índice de privación multidimensional (IPM) para el Brasil y los estados brasileños en 2003 y 2008. La construcción del índice se inspiró en la metodología desarrollada por Alkire y Santos (2010) y se basa en cuatro dimensiones (condiciones de vivienda, salud, niveles de educación y participación en el mercado de trabajo) y 13 indicadores. A partir del IPM se analizó la privación desde distintas perspectivas, en procura de una visión más completa de la privación en el Brasil urbano según un enfoque multidimensional.

Los resultados revelaron que la mayoría de las personas viven en hogares sin privación no monetaria o con privación relativa a un solo indicador. Los hogares con carencias en muchos indicadores corresponden a una pequeña proporción de la muestra. Asimismo, el número relativo de personas en hogares con privación disminuyó y, entre aquellos que ya se encontraban en condiciones de privación, la proporción en un único indicador aumentó.

Las regularidades y relaciones entre los indicadores se examinaron mediante un análisis factorial. Algunos aspectos de la privación pueden manifestarse en grupos de dos o más indicadores, como por ejemplo: agua y alcantarillado, trabajo infantil e inasistencia escolar y presencia de activos, adultos sin trabajo y analfabetos funcionales en el hogar. Los resultados sugieren que las políticas para reducir la privación multidimensional también pueden orientarse a las sinergias que existan entre las diferentes dimensiones.

A continuación se comparó la privación medida según los ingresos con la privación multidimensional en los estados brasileños y se confirmó la desigualdad regional de acuerdo con las dos medidas. Cabe destacar que, tanto la privación monetaria como la privación multidimensional disminuyeron de manera considerable en la gran mayoría de los estados. Empero, se observó una escasa correlación entre los indicadores de privación monetaria y no monetaria.

Por último, se estudió la dinámica de la privación mediante cohortes sintéticas y modelos de MCo. De ese modo, fue posible analizar la relación entre atributos como sexo, raza, año de nacimiento y educación del jefe de hogar con la propensión de esa unidad a caer o permanecer en situación de privación.

La privación, tanto según la medida monetaria como no monetaria, afecta de manera más significativa a los hogares encabezados por personas de raza negra, mujeres, personas sin calificación y jóvenes, incluso después del control mediante las demás variables. Se observaron también dinámicas de evolución temporal diferentes para los diversos indicadores.

Los resultados muestran que el aumento del nivel de escolaridad reduce tanto la privación monetaria como la no monetaria. Sin embargo, la educación pasó a ser menos efectiva para ese primer indicador. Esto puede 
explicarse en parte por el incremento general de los niveles educativos y la disminución de la desigualdad de ingresos en el Brasil.

No obstante, el resultado relativo a las cohortes no es neutro como la variable dependiente considerada. En el caso de la privación monetaria la tendencia es clara: la propensión a la privación es menor entre las cohortes de más edad. Este resultado puede reflejar una peor inserción de las generaciones más jóvenes en el mercado de trabajo.

En cuanto a la privación en por lo menos un indicador, pese a que la edad tiene un efecto en el tipo de privación, no se aprecian grandes diferencias respecto de las personas con privación no monetaria. Este tema podría ser objeto de futuras investigaciones. Por otra parte, al analizar la privación en por lo menos dos indicadores, se registran valores más elevados entre las cohortes de edad más avanzada, que sugieren una mayor incidencia de la privación multidimensional entre los individuos de mayor edad. Estas cohortes tienden a enfrentar algunos problemas específicos de privación multidimensional que también podrían investigarse con mayor profundidad.

Las pruebas recogidas en este trabajo indican que la medida multidimensional no monetaria puede comprender aspectos relacionados con la privación y el bienestar que no es posible aprehender con la medida monetaria. Por ejemplo, un hogar clasificado como en situación de privación según los patrones de ingresos puede no ser considerado en esa condición de acuerdo con otros enfoques, como el de privación multidimensional. La privación, definida como insuficiencia de bienestar, debe estudiarse mediante variables monetarias y no monetarias (Bourguignon y Chakravarty, 2003; Thorbecke, 2005). Con este trabajo también se procuró avanzar en el entendimiento de la privación en el Brasil urbano desde una perspectiva multidimensional y sugerir algunos temas para investigaciones futuras.

ANEXO

Como se muestra en el mapa A.1, el Brasil es uno de los mayores países del mundo, con más de 8 millones de kilómetros cuadrados. Se divide en cinco macrorregiones, a saber: norte, nordeste, sudeste, sur y centro-oeste, y 26 estados y el Distrito Federal.

MAPA A.1

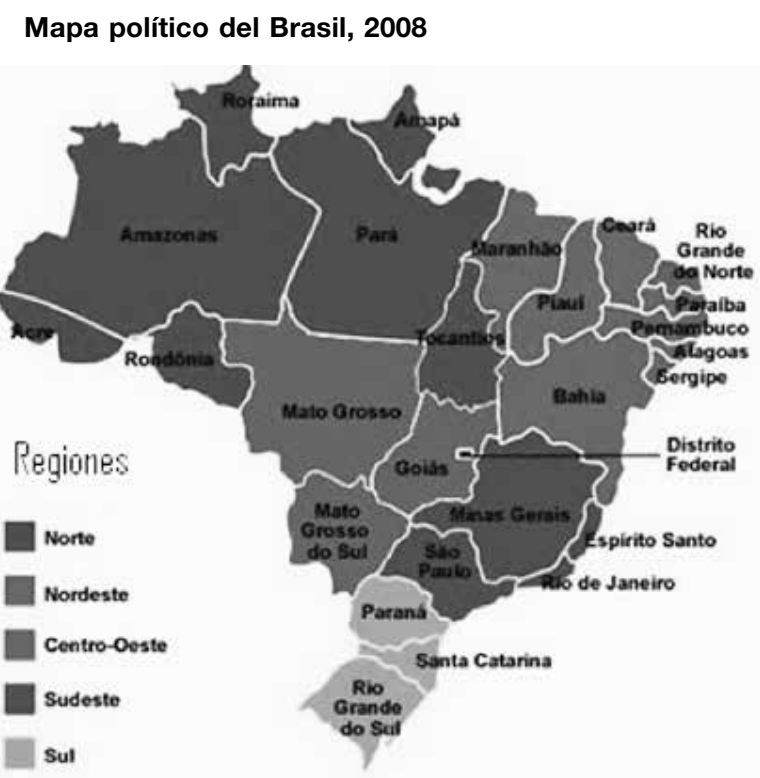

Fuente: http://www.brasil-turismo.com/geografia.htm 


\section{Bibliografía}

Alkire, S. y J. Foster (2007), "Counting and multidimensional poverty measurement", OPHI Working Paper, $\mathrm{N}^{\circ} 32$, Oxford, Oxford Poverty and Human Development Initiative [en línea] http:// www.ophi.org.uk/wp-content/uploads/OPHI-wp32.pdf.

Alkire, S. y M. Santos (2010), "Acute multidimensional poverty: a new index for developing countries", Human Development Research Papers, $\mathrm{N}^{\circ}$ 2010/11, Nueva York, Oficina encargada del Informe sobre Desarrollo Humano, Programa de las Naciones Unidas para el Desarrollo.

Atkinson, A. (2003), "Multidimensional deprivation: contrasting social welfare and counting approaches", Journal of Economic Inequality, vol. $1, \mathrm{~N}^{\circ} 1$, Springer.

Bagolin, I. y R. Ávila (2006), "Poverty distribution song the Brazilian states: a multidimensional analysis using capabilities and needs approaches", Anais do XXXIV Encontro Nacional de Economia, Río de Janeiro, Asociación Nacional de Centros de Postgrado en Economía.

Banco Mundial (2006), "Brazil: measuring poverty using household consumption", Documento del Banco Mundial, $\mathrm{N}^{\circ} 36358-\mathrm{BR}$, Washington, D.C.

Baulch, B. y E. Masset (2003), "Do monetary and non-monetary indicators tell the same story about chronic poverty? A study of Vietnam in the 1990s", World Development, vol. 31, N 3, Amsterdam, Elsevier.

Ben-Schlomo, Y. y D. Kuh (2002), "A life course approach to chronic disease epidemiology: conceptual models, empirical challenges, and interdisciplinary perspectives", International Journal of Epidemiology, vol. 31, N2 2, Oxford, Oxford University Press.

Bourguignon, F. y S. Chakravarty (2003), "The measurement of multidimensional poverty", Journal of Economic Inequality, vol. $1, \mathrm{~N}^{\circ} 1$, Springer.

D'Ambrosio, C. y R. Rodrigues (2008), "Deprivation in the São Paulo districts: evidence from 2000", World Development, vol. 36, $\mathrm{N}^{\circ} 6$, Amsterdam, Elsevier.

Decancq, K. y M. Lugo (2010), "Weights in multidimensional indices of well-being: an overview", Center for Economic Studies Discussions Papers, $\mathrm{N}^{\circ}$ CES10.06, Lovaina, Universidad Católica de Lovaina.

Ferreira, F. y A. Lugo (2012), "Multidimensional poverty analysis: looking for a middle ground", Policy Research Working Paper, $\mathrm{N}^{\circ}$ 5964, Washington, D.C., Banco Mundial.

Golgher, A.B. (2010a), "Perceived deprivation in Brazil in a multidimensional perspective", Revue d'économie régionale et urbaine, $\mathrm{N}^{\circ} 2$, París, Editions NecPlus.

(2010b), "Multidimensional poverty in Brazil: income, assets and expenses", documento presentado en la 57th Annual North American Meetings of the Regional Science Association International, Denver.

(2009), "Poverty in Brazil: income, material hardship and the perception of deprivation", documento presentado en la reunión anual de la Population Association of America, Detroit.

IBGE (Instituto Brasileño de Geografia y Estadística) (2008), Pesquisa Nacional por Amostra de Domicílios 2008, Río de Janeiro.

(2003), Pesquisa Nacional por Amostra de Domicílios 2003, Río de Janeiro.

Lopes, H., P. Macedo y A. Machado (2004), "Análise multidimensional de privación para as meso e microrregiões de Minas Gerais" [en línea] http://www.cedeplar.ufmg.br/diamantina2004/textos/ D04A014.PDF.

(2003), "Indicador de privación: aplicação de uma abordagem multidimensional ao caso brasileiro", Textos para discussão, $\mathrm{N}^{\circ} 223$, Belo Horizonte, Centro de Desarrollo y Planificación Regional (CEDEPLAR).

Markunsen, A. (2006), "Urban development and the politics of a creative class: evidence from the study of artists", Environment and Planning A, vol. 38, $\mathrm{N}^{\circ} 10$, Londres, Pion.

Neri, M. (2008), "A perceived human development index", documento presentado en el XVI Encuentro Nacional de Estudios Poblacionales, Asociación Brasileña de Estudios Poblacionales (ABEP).

Noronha, K.M.S. (2005), "A relação entre o Estado de saúde e a desigualdade de renda no Brasil", tesis, Belo Horizonte, Centro de Desarrollo y Planificación Regional (CEDEPLAR)/Universidad Federal de Minas Gerais (UFMG).

Ribas, R., A. Machado y A. Golgher (2011), "Flutuações e persistência na privación: uma análise de decomposição transitória-crônica para o Brasil", Pesquisa e Planejamento Econômico, vol. 41, $\mathrm{N}^{\circ} 2$, Río de Janeiro, Instituto de Investigación Económica Aplicada (IPEA).

Reither, E., R. Hauser y Y. Yang (2009), "Do birth cohorts matter? Age-period-cohort analyses of the obesity epidemic in the U.S.", Social Science and Medicine, vol. 69, N 10, Amsterdam, Elsevier.

Rocha, S. (2003), Privación no Brasil: afinal, de que se trata?, Río de Janeiro, Editora FGV.

Rocha, L., R. Moreira y G. Santos (2008), "Índice de desenvolvimento da familia (IDF): uma análise para as microrregiões e grupos demográficos do estado de Minas Gerais", Anais do XVIII Seminário sobre a Economia Mineira [Proceedings of the 18th Seminar about Economy in Minas Gerais], Belo Horizonte, Universidad Federal de Minas Gerais.

Sen, A. (2001), Desigualdade reexaminada, São Paulo, Record.

(1999), Development as Freedom, Oxford, Oxford University Press.

Silva, M. y R. Barros (2006), "Privación multidimensional no Brasil", Anais do XXXIV Encontro Nacional de Economia [Proceedings of the 34th Brazilian Economics Meeting], Río de Janeiro, Asociación Nacional de Centros de Postgrado en Economía.

Thorbecke, E. (2005), "Multi-dimensional poverty: conceptual and measurement issues, Brasilia", documento presentado en la Conferencia internacional "The many dimensions of poverty", Brasilia, Centro Internacional de la Privación.

Yang, Y. (2008a), "Trends in U.S. adult chronic disease mortality 1960-1999: age, period, and cohort variations", Demography, vol. $45, \mathrm{~N}^{\circ} 2$, Springer.

(2008b), "Social inequalities in happiness in the U.S. 19722004: an age-period-cohort analysis", American Sociological Review, $\mathrm{N}^{\circ} 73$, Sage.

(2007), "Is old age depressing? Growth trajectories and cohort variations in late- life depression", Journal of Health and Social Behavior, vol. 48, $\mathrm{N}^{\circ} 1$, Sage.

Yang, Y. y L. Lee (2009), "Sex and race disparities in health: cohort variations in life course patterns", Social Forces, vol. 87, $\mathrm{N}^{\circ} 4$, Oxford, Oxford University Press. 